\title{
Subglottic Chondrosarcoma Presenting Only Mild Acute-Onset Dyspnea: A Case Report and Review of the Literature
}

\author{
Su-wei Tsai Chun-yen Ou \\ Department of Otolaryngology, College of Medicine and Medical Center, National Cheng \\ Kung University, Tainan, Taiwan, ROC
}

\section{Key Words}

Chondrosarcoma $\cdot$ Subglottic chondrosarcoma

\begin{abstract}
Chondrosarcoma is categorized as a malignant cartilaginous tumor, which occurs rarely in the craniofacial region. We report the case of a 68-year-old man with chondrosarcoma in the subglottic area. His chief symptoms were hoarseness and mild dysphagia. A computed tomography scan revealed a lesion with expansion of the cricoid cartilage and marked reduction of the airway. After biopsy, histological inspection showed that chondrocytes are multi-nucleus, their size does not differ much and mitosis is not obvious. These are all characteristics of a low-grade chondrosarcoma. We performed an organ-preserving operation by debulking the low-grade malignant tumor in order to keep a patent airway. No further metastasis or airway compromise was evident during the 1-year follow-up visit.
\end{abstract}

(C) 2014 S. Karger AG, Basel

\section{Introduction}

Chondrosarcoma of the larynx is quite rare, comprising $1 \%$ of all laryngeal primary tumors [1-3]. Primary malignant lesions originating from the subglottis area are also rare and constitute between 1 and $3.6 \%$ of all laryngeal cancers [4-6]. In subglottic cancers, epidermoid carcinoma was found in $71.8 \%$ of the cases, the second most common type was chondrosarcoma in $10.3 \%$ of the cases [5]. Definite incidences are unknown and difficult to assess, as low-grade chondrosarcomas are frequently misinterpreted as chondromas.

Chun-yen Ou, MD

Department of Otolaryngology, College of Medicine and Medical Center

National Cheng Kung University

Tainan, Taiwan (ROC)

E-Mail n045348@mail.hosp.ncku.edu.tw 
Tsai et al.: Subglottic Chondrosarcoma Presenting Only Mild Acute-Onset Dyspnea: A

Although surgical excision is still the mainstay of treatment, the operation extent for this relative slow progression cancer is still equivocal. In this article, we present a case of subglottic chondrosarcoma and a review of the literature.

\section{Case Report}

A 68-year-old Asian male presented with a 1-year history of hoarseness, mild dysphagia, and a 1-month history of dyspnea. On examination, the patient had no stridor. Transnasal flexible endoscopy (fig. 1) revealed a smooth mass below the glottis distorting the larynx. Half of the subglottic area was obstructed, and he also had left vocal cord paresis. A computed tomography (CT) scan of the neck revealed a solid mass in the supraglottic region, extending from the cricoid cartilage to the left thyroid cartilage area, with no neck lymphadenopathy (fig. 2). The CT scan showed gross expansion of the cricoid cartilage with marked reduction of the airway, but the overlying mucosa was smooth. Some intermittent calcification spots within the extending tumor mass were also noted. On admission, the patient received laryngomicrosurgery for biopsy and tracheostomy to secure the airway. Multiple biopsies were taken which revealed features consistent with low-grade chondrosarcoma (fig. 3c). As the patient is running a company, he needs to talk frequently. In agreement with the patient and his family, and considering the low-grade nature of the tumor, endoscopic debulking was decided. As a result, we performed a laryngomicrosurgery operation. The subglottic lesion was debulked with electrocauterization to widen the compromised airway. Histologic diagnosis revealed a low-grade chondrosarcoma of the cricoid. The patient was well after removal of the tracheostomy tube 2 months later. During the 12-month follow-up, the lesion did not increase in size and no metastasis was detected on the CT image. The patient receives ambulatory treatment and is doing well, and transnasal flexible endoscopy revealed that his airway is relatively patent (fig. 4).

\section{Discussion}

Cartilaginous tumors of the larynx were first described by Heusinger in 1822. The incidence of laryngeal chondrosarcoma has been underreported, as differentiation between chondroma and chondrosarcoma has been a topic of debate. Chondrosarcoma is well known as the fourth most common tumor of the bone and tend to be bulky, lobular lesions that can be seen anywhere, although with a preponderance in the lower appendicular skeleton and the pelvis. Compared to chondrosarcomas in other systems, only $2-10 \%$ of all chondrosarcomas arise in the head and neck, with the majority encountered in the maxilla. However, laryngeal chondrosarcomas, first described in the early 1800s, are rare tumors of the head and neck, accounting for $<0.2 \%$ of all head and neck malignancies and up to $1 \%$ of all laryngeal tumors $[1,7]$. The most common malignant neoplasm arising in the subglottis area is epidermoid carcinoma, which comprised 55\% of tumors in one review [5]. Adenoid cystic carcinoma is the second most common type, followed by chondrosarcoma. In one study, $71.8 \%$ of the patients had epidermoid cancer and $10.3 \%$ had chondrosarcoma [5]. Patients with subglottic lesions are suffering from a tumor that slowly but progressively encroaches on the glottic lumen and causes hoarseness, airway obstruction or difficulty in breathing, a mass lesion, dysphagia or dysphonia, stridor or pain. Many patients had more than one symptom at the time of initial presentation, but hoarseness was the most frequently identified symptom in $64.9 \%$ of the patients. The other complaints depend upon how quickly 
Tsai et al.: Subglottic Chondrosarcoma Presenting Only Mild Acute-Onset Dyspnea: A

the lesion develops and the anatomic location of the primary tumor [1]. The age of presentation varies between the fifth to seventh decades, the average age being 66 years. The tumor is seven times more common in Caucasians, and three times more common in men compared to women [7]. There is no significant difference in overall survival between genders. There was a significant decrease in overall survival for patients who were older than 60 years at initial presentation than those who were younger which may be accounted for by the advanced age of patients in general medical condition [1]. Lesions arising from the cricoid cartilage usually present with dyspnea, hoarseness and dysphagia [7]. The symptoms present for a mean duration of 28.2 months. The majority of tumors involved the cricoid cartilage with a mean size of $3.5 \mathrm{~cm}$ [1]. Some authors believe that idiopathic vocal fold paralysis is an exclusive sign of cricoid chondrosarcoma and may be related to the involvement of recurrent laryngeal nerve or cricoarytenoid joint fixation. Thyroid cartilage chondrosarcomas may present as an asymptomatic neck mass [7]. Furthermore, the overall size of the lesion did not significantly affect the overall outcome.

The majority of lesions were presented as multiple, irregular fragments of bone and soft tissue. Areas of ossification in hyaline cartilages show a predilection for the development of laryngeal chondrosarcomas [1]. Laryngeal chondrosarcomas usually arise from the posterior lamina of the cricoid cartilage $(78 \%)$ or the thyroid cartilage (18\%), and rarely from the arytenoids and epiglottis [3]. Signs and symptoms depend on the location of the tumor. Lesions arising from the cricoid cartilage usually present with dyspnea, hoarseness and dysphagia. In the larynx, chondrosarcomas typically present with slow growth, rare metastasis and low recurrence rates. Chondrosarcoma causes local destruction due to a mass effect before it invades the surrounding tissues. Radiologically, this tumor can present as a lesion of variable density, showing either endolaryngeal spread (growing inwards, confined by the outer margin of the cartilage of origin) or extralaryngeal spread (growth extending beyond the confines of the larynx into the surrounding soft tissue). On CT scanning, chondrosarcomas demonstrate fine, punctuate, and stippled to coarse calcification within the tumor $[1,8]$.

The diagnosis of chondrosarcoma is based on criteria devised by Lichtenstein and Joffe for malignant cartilaginous tumors of bony origin, published in 1943 (see Friedlander and Lyons [6]). The grading system for chondrosarcomas was further developed by Evans et al. [9]. When combined with patient outcome to yield a declining survival for higher-grade tumors, a 5-year survival of 90,81 , and $43 \%$ for grades I, II, and III, respectively, was reported. In the axial skeleton, mostly referred to as CAMMC (chondrosarcoma or chondrosarcoma with additional malignant mesenchymal component), the median survival is of 6 months with an approximate $70 \%$ chance of metastases, usually to the lung or soft tissues [9-11]. In sharp contrast to CAMMC of the axial skeleton, patients with laryngeal CAMMC have a more favorable long-term prognosis, although a similar recurrence/metastatic potential is reported with a local recurrence rate of $62 \%$ for laryngeal CAMMC.

Depending on the degree of differentiation, chondrosarcomas are classified as low-, medium- or high-grade tumors. Most of the reported chondrosarcomas of the larynx are low grade, although some high-grade tumors have been described. The definitive treatment of laryngeal chondrosarcoma has been a topic of debate. Preservation of laryngeal function with surgical eradication of the neoplasm is the preferred option [12-14]. However, according to Thome and colleagues, total resection of cricoid cartilage with thyrotracheal anastomosis over a stent is an alternative technique to total laryngectomy in patients with chondrosarcoma (see Finn et al. [12]). Neck dissections should include pretracheal, paratracheal, prelaryngeal or precricoid (Delphian) nodes, lower and middle jugular nodes, 


\begin{tabular}{l|l}
\hline DOI: $10.1159 / 000358381$ & $\begin{array}{l}\text { C 2014 S. Karger AG, Basel } \\
\text { www.karger.com/cro }\end{array}$ \\
\hline
\end{tabular}

Tsai et al.: Subglottic Chondrosarcoma Presenting Only Mild Acute-Onset Dyspnea: A Case Report and Review of the Literature

and supraclavicular nodes. Patients treated with combined treatment (surgery and radiotherapy) have the highest disease-free survival.

\section{Conclusion}

Laryngeal chondrosarcomas are rare tumors commonly occurring in men in their seventh decade. The cricoid cartilage is commonly involved. Surgery is the principal treatment modality. However, a conservative approach to management of these tumors should be borne in mind, especially in patients with associated comorbid conditions.

\section{Disclosure Statement}

The authors declare that they have no competing interests.

\section{References}

1 Thompson LD, Gannon FH: Chondrosarcoma of the larynx: a clinicopathologic study of 111 cases with a review of the literature. Am J Surg Pathol 2002;26:836-851.

-2 Burkey BB, Hoffman HT, Baker SR, Thornton AF, McClatchey KD: Chondrosarcoma of the head and neck. Laryngoscope 1990;100:1301-1305.

3 Jackson RS, Leon ME, McCaffrey TV: Chondrosarcoma of the Subglottic Larynx: Submucosal Microdissection With the Operating Microscope. Laryngoscope 2013;123:1216-1219.

4 Oestreicher-Kedem Y, Dray TG, Damrose EJ: Endoscopic resection of low grade, subglottic chondrosarcoma. J Laryngol Otol 2009;123:1364-1366.

5 Dahm JD, Sessions DG, Paniello RC, Harvey J: Primary subglottic cancer. Laryngoscope 1998;108:741-746.

-6 Friedlander PL, Lyons GD: Chondrosarcoma of the larynx. Otolaryngol Head Neck Surg 2000;122:617.

7 Bathala S, Berry S, Evans RA, Brodie S, Altaan O: Chondrosarcoma of larynx: review of literature and clinical experience. J Laryngol Otol 2008;122:1127-1129.

-8 Policarpo M, Taranto F, Aina E, Aluffi PV, Pia F: Chondrosarcoma of the larynx: a case report. Acta Otorhinolaryngol Ital 2008;28:38-41.

-9 Evans HL, Ayala AG, Romsdahl MM: Prognostic factors in chondrosarcoma of bone: a clinicopathologic analysis with emphasis on histologic grading. Cancer 1977;40:818-831.

10 Nakayama M, Brandenburg JH, Hafez GR: Dedifferentiated chondrosarcoma of the larynx with regional and distant metastases. Ann Otol Rhinol Laryngol 1993;102:785-791.

11 Bleiweiss IJ, Kaneko M: Chondrosarcoma of the larynx with additional malignant mesenchymal component (dedifferentiated chondrosarcoma). Am J Surg Pathol 1988;12:314-320.

12 Finn DG, Goepfert H, Batsakis JG: Chondrosarcoma of the head and neck. Laryngoscope 1984;94:1539-1544.

-13 Neel HB, 3rd, Unni KK: Cartilaginous tumors of the larynx: a series of 33 patients. Otolaryngol Head Neck Surg 1982;90:201-207.

14 Lavertu P, Tucker HM: Chondrosarcoma of the larynx. Case report and management philosophy. Ann Otol Rhinol Laryngol 1984;93:452-456. 


\section{Case Reports in Oncology}

\begin{tabular}{l|l}
\hline Case Rep Oncol 2014;7:86-91 & \\
\hline DOI: $10.1159 / 000358381$ & $\begin{array}{l}\text { C 2014 S. Karger AG, Basel } \\
\text { www.karger.com/cro }\end{array}$ \\
\hline
\end{tabular}

Tsai et al:: Subglottic Chondrosarcoma Presenting Only Mild Acute-Onset Dyspnea: A Case Report and Review of the Literature

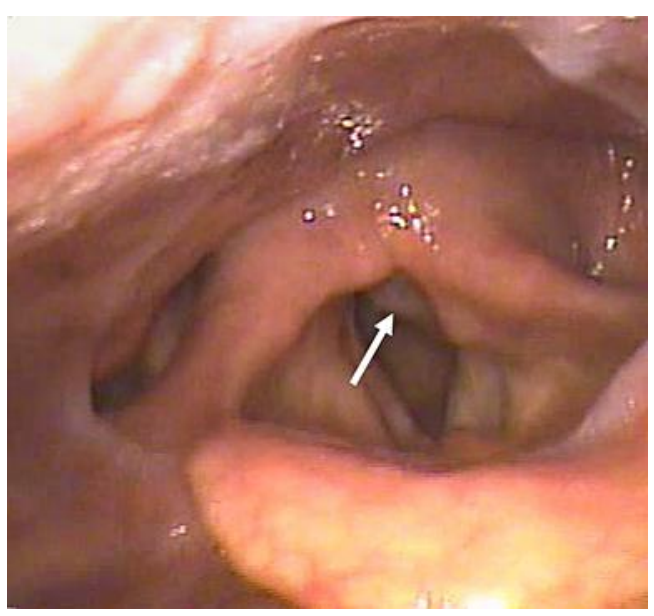

Fig. 1. The white arrow indicates the bulging lesion in the subglottic area on the left side of the larynx and the lesion involving the left arytenoid, which is part of the arytenoid-epiglottic fold causing the left vocal cord palsy.

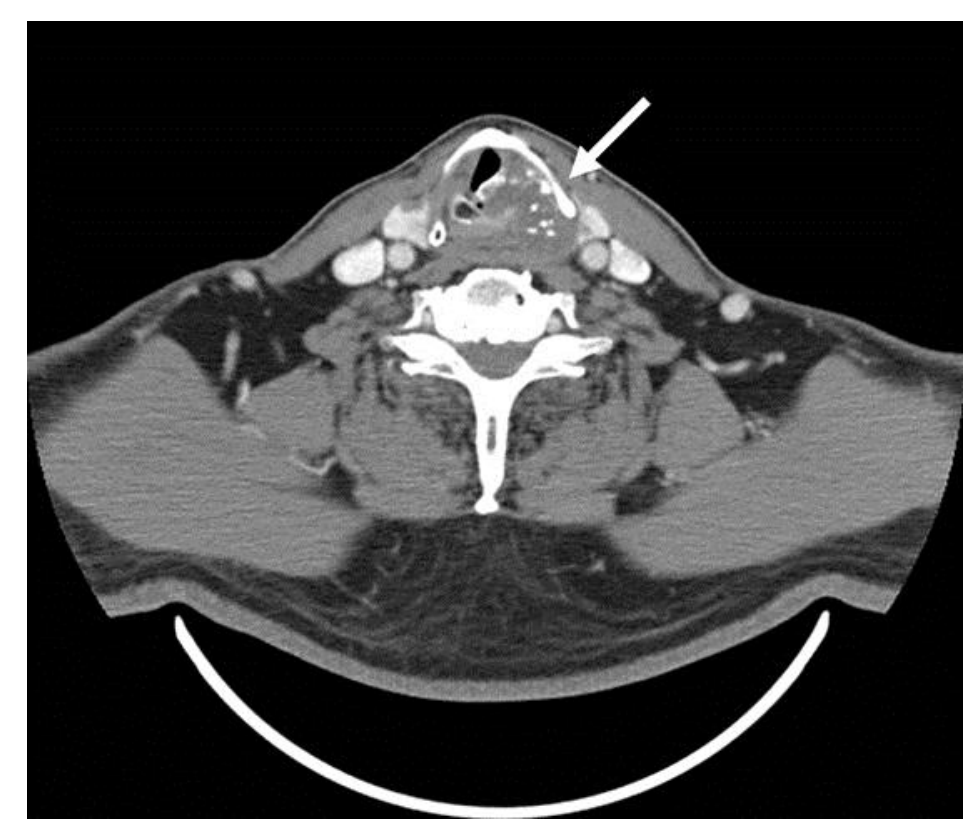

Fig. 2. The white arrow indicates the heterogeneous lesion and the radiopaque spots representing the calcified lesion and suggesting cartilage or a bony nature. 
Tsai et al.: Subglottic Chondrosarcoma Presenting Only Mild Acute-Onset Dyspnea: A Case Report and Review of the Literature
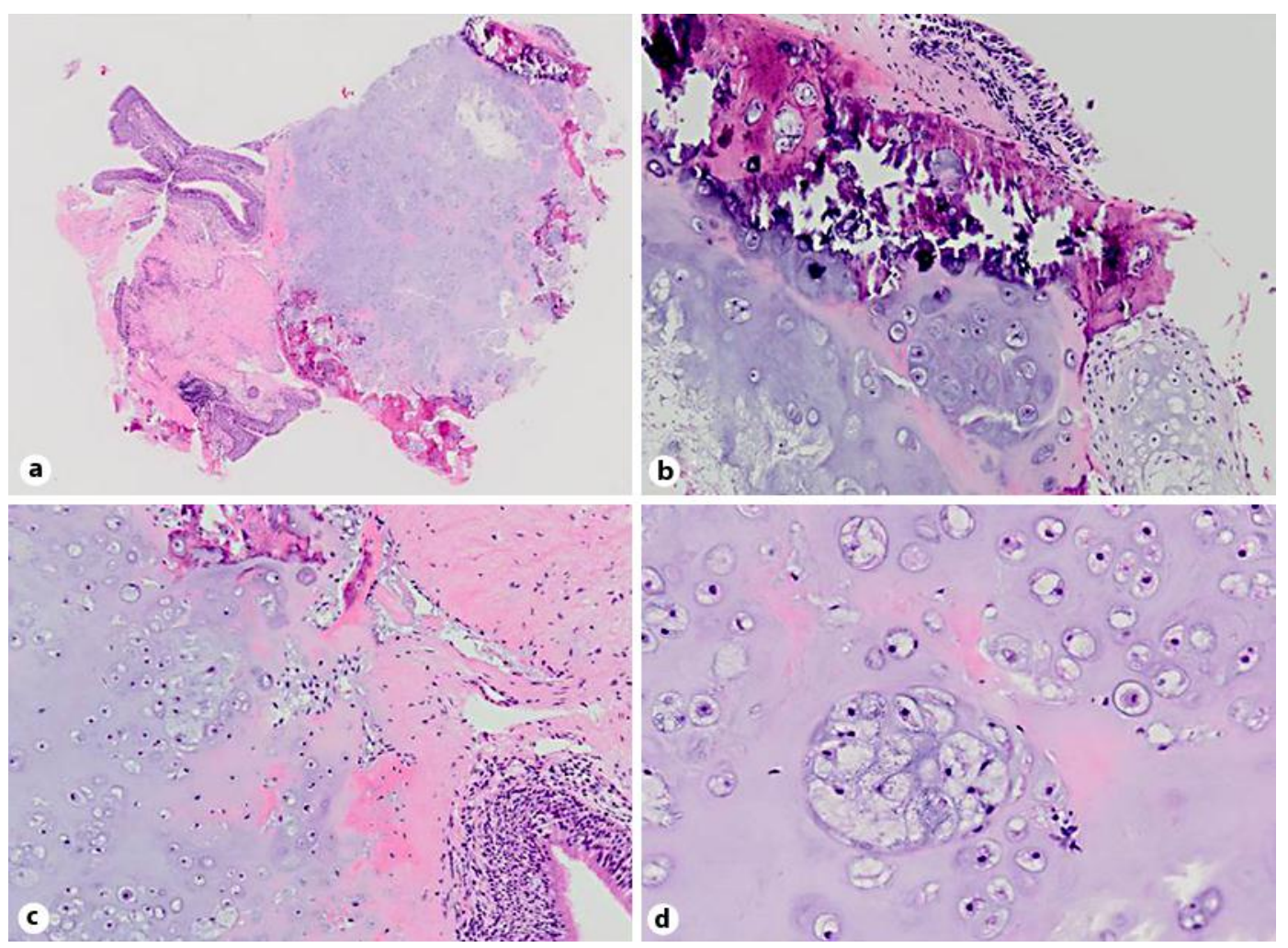

Fig. 3. a The infiltrated margin is showing a tumor beneath the epithelium and a normal mucosa with ciliated respiratory epithelium and squamous epithelium, HE stain, $40 \times$. b The chondrocyte is multinucleus, the size does not differ much and mitosis is not obvious, which are all characteristics of lowgrade chondrosarcoma, 200×. c Chondroid mixoid change representing the primitive proliferation along with the spindle cells, $100 \times$. $\mathbf{d}$ Multiple cell nuclei in one lacuna and mitosis are shown, $200 \times$.

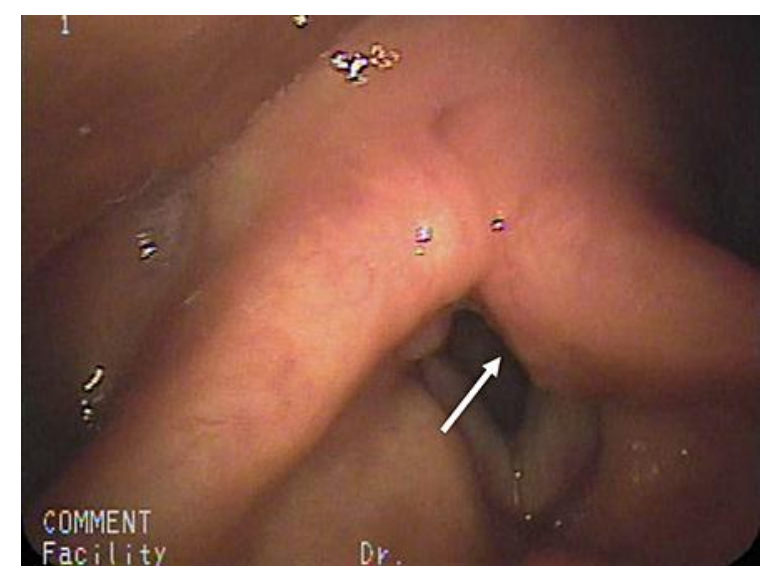

Fig. 4. The subglottic lesion can hardly be seen in the subglottic area with forced inhalation. 